positive patients may have contracted the virus from another source. Moreover, we did not examine the risk of COVID-19 in the outpatient setting. Nevertheless, this result is reassuring and should encourage timely treatment of healthcare problems, even during the COVID-19 pandemic, to avoid unnecessary complications.

Acknowledgments. Data for the study were provided by the Arkansas Clinical Data Repository (AR-CDR) maintained by the Department of Biomedical Informatics in the College of Medicine at the University of Arkansas for Medical Sciences (UAMS). The AR-CDR is approved to operate as an enterprise data resource to support research across UAMS.

Financial support. No financial support was provided relevant to this article.

Conflicts of interest. B.R. received a consulting fee from Alexion pharmaceutical. All other authors report no conflicts of interest.

\section{References}

1. Brainin M. Stroke care and the COVID19 pandemic words from our president. World Stroke Organization website. www.world-stroke.org/ news-and-blog/news/stroke-care-and-the-covid19-pandemic. Accessed October 5, 2020.

2. Onteddu SR, Nalleballe K, Sharma R, Brown AT. Underutilization of healthcare for strokes during the COVID-19 outbreak. Int J Stroke 2020. doi: 10. $1177 / 1747493020934362$.

3. Truffa Giachet A, Barbero U, Ugo F, et al. Reduced rate of hospital admissions for ACS during COVID-19 outbreak in northern Italy. $N$ Engl J Med 2020;383:88-89.

4. CDC COVID data tracker. Centers for Disease Control and Prevention website. https://www.cdc.gov/coronavirus/2019-ncov/cases-updates/us-casesdeaths.html. Accessed October 5, 2020.

5. US and world population clock. US Census Bureau website. https://www. census.gov/popclock/. Accessed October 5, 2020.

\title{
Prolonged incubation of severe acute respiratory syndrome coronavirus 2 (SARS-CoV-2) in a patient on rituximab therapy
}

\author{
Alan G. Koff MBBS ${ }^{1, a}$ (1) , Maudry Laurent-Rolle MD, PhD 2,a (1), Jack Chun-Chieh Hsu PhD ${ }^{3}$ and Maricar Malinis $\mathrm{MD}^{2}$ (1) \\ ${ }^{1}$ Section of Infectious Diseases, Department of Internal Medicine, University of California Davis, Davis, California, ${ }^{2}$ Section of Infectious Diseases, Department of \\ Internal Medicine, Yale University School of Medicine, New Haven, Connecticut and ${ }^{3}$ Departments of Immunobiology and Cell Biology, Yale University School of \\ Medicine, New Haven, Connecticut
}

\begin{abstract}
The incubation period of severe acute respiratory syndrome coronavirus 2 (SARS-CoV-2) is rarely $>14$ days. We report a patient with hypogammaglobulinemia who developed coronavirus disease 2019 (COVID-19) with a confirmed incubation period of at least 21 days. These findings raise concern for a prolonged presymptomatic transmission phase, necessitating a longer quarantine duration in this patient population.
\end{abstract}

Severe acute respiratory syndrome coronavirus 2 (SARS-CoV-2) was discovered in Wuhan, China, and has since become a global pandemic through person-to-person spread. SARS-CoV-2 exhibits presymptomatic transmission during the incubation period, where an individual is contagious prior to symptom onset. ${ }^{1}$ Defining the incubation period, therefore, has infection control and public health implications because a longer incubation necessitates a longer quarantine duration after an exposure.

Mean incubation periods range from 5.0 to 7.2 days, and a median incubation period of 5.1 days has been reported. ${ }^{2-6}$ In 2 studies, the 95th percentiles of the distribution were reported as 12.5 days and 13 days, and another 3 studies reported the 99th percentile as 11.9 days, 14 days, and 14.9 days, respectively. ${ }^{2-4,6,7}$ In the vast majority of cases, the incubation period is far less than 14 days, which has helped to inform the Centers for Disease Control and Prevention (CDC) recommendations for a 14-day quarantine period after a known coronavirus disease 2019 (COVID-19) exposure. ${ }^{8}$ However, these cases represent the general

Author for correspondence: Alan G. Koff, E-mail: akoff@ucdavis.edu

a Authors of equal contribution.

Cite this article: Koff AG, et al. (2021). Prolonged incubation of severe acute respiratory syndrome coronavirus 2 (SARS-CoV-2) in a patient on rituximab therapy. Infection Control \& Hospital Epidemiology, 42: 1286-1288, https://doi.org/10.1017/ice.2020.1239 population and do not provide detailed information on subpopulations in whom the incubation period may differ. Herein, we present a case with objectively confirmed COVID-19 with a prolonged incubation period proven through viral culture.

\section{Case presentation}

A 71-year-old female on rituximab for granulomatosis with polyangiitis presented with shortness of breath and nonproductive cough. Six weeks prior to admission, several family members had been diagnosed with COVID-19 infection, prompting her to undergo testing despite being asymptomatic. Her nasopharyngeal (NP) swab polymerase chain reaction (PCR) test for SARS-CoV-2 was positive. She was self-isolating, and her only contact was a family member who had recovered from mild COVID-19 illness and had since been asymptomatic. Repeat NP PCR testing 13 days later was also positive. On day 21 after the first test, the patient developed progressive dyspnea on exertion, a minimally productive cough, significant fatigue, and nonbloody diarrhea.

She was admitted to hospital on day 36 after her first test. She was febrile to $38.8^{\circ} \mathrm{C}$ and her oxygen saturation was $93 \%$ on room air. She was placed on $2 \mathrm{~L} /$ minute of supplemental oxygen. Computed tomography (CT) of the chest demonstrated bilateral

(c) The Author(s), 2020. Published by Cambridge University Press on behalf of The Society for Healthcare Epidemiology of America. This is an Open Access article, distributed under the terms of the Creative Commons Attribution licence (http://creativecommons.org/licenses/by/4.0/), which permits unrestricted re-use, distribution, and reproduction in any medium, provided the original work is properly cited. 
(A)

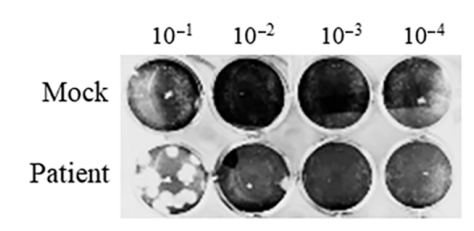

(B)

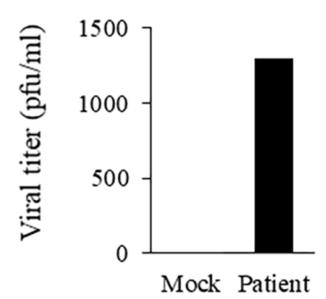

(C)

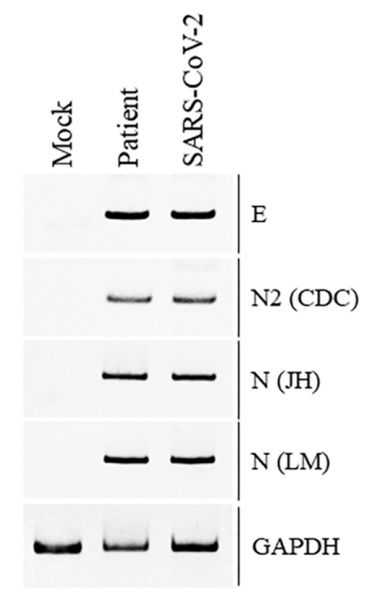

(D)

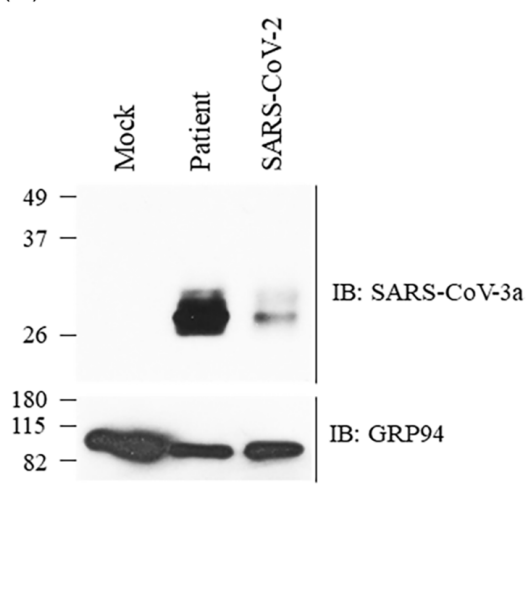

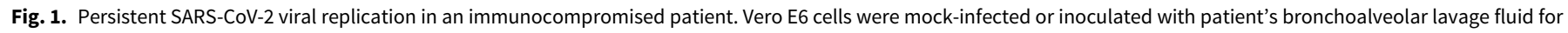

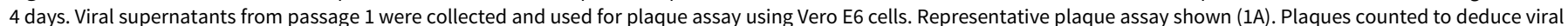

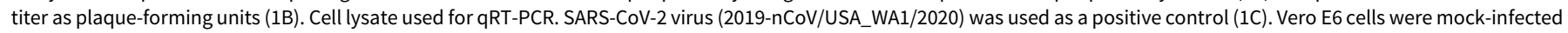

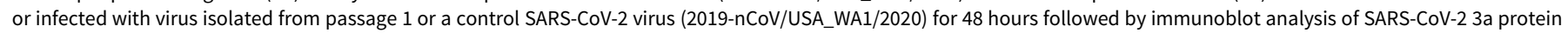
using an antibody against SARS-CoV $3 a$ (1D). The primer sequences can be found in Supplementary Table 1 (online).

peribronchovascular ground-glass opacities (Supplementary Fig. 1 online). Relative to the day of her first test, she had repeat SARS-CoV-2 NP PCR tests on days 36, 37, and 40, which were negative. Serology for SARS-CoV-2 was negative. Flow cytometry of peripheral blood demonstrated no circulating B-cells, and an immunoglobulin panel demonstrated low levels of IgM, IgG, and IgA consistent with a history of receiving rituximab. Bronchoalveolar lavage (BAL) on hospital day 5 revealed a positive SARS-CoV-2 PCR with N1 and N2 cycle thresholds of 29 and 28, respectively. The patient was weaned off supplemental oxygen and was discharged on hospital day 9.

The patient's BAL fluid was stored at $-80^{\circ} \mathrm{C}$ then thawed and inoculated into Vero E6 cell culture. Viral supernatant was harvested on day 4 after inoculation for plaque assay demonstrating infectious virus with a titer of $1.3 \times 10^{3} \mathrm{pfu} / \mathrm{mL}$ on passage 1 (Fig. 1A and 1B). Nucleic acid extraction from the cell lysate confirmed the presence of SARS-CoV-2 by reverse-transcription realtime PCR and by polyacrylamide gel (Fig. 1C). Isolate from the first passage of the BAL specimen was used to infect Vero E6 cells for 48 hours. Cell lysates were probed for protein analysis using an antibody raised against SARS-CoV 3a antibody which demonstrated bands consistent with SARS-CoV-2 3a protein (Fig. 1D). These studies indicate that infectious SARS-CoV-2 virus was isolated from the patient's BAL.

\section{Discussion}

This case demonstrates an objectively confirmed asymptomatic SARS-CoV-2 infection with symptom onset 21 days after her positive test. Furthermore, since an NP PCR can be falsely negative on the first day of infection, her incubation period may have been even greater. ${ }^{9}$ Lower respiratory tract sampling demonstrated viable SARS-CoV-2 virus, though the NP PCR was negative. A prior study demonstrated that NP PCR had a false negative rate of $66 \%$ by day 21 , which may explain our observation. ${ }^{9}$

Reports of incubation periods $>21$ days are very rare. A patient with an incubation period of 24 days was reported; however, the incubation period was defined as the time between the earliest potential date of exposure to the first day of symptom onset, potentially leading to overestimation. ${ }^{6}$ A case report described a patient with an incubation period of at least 38 days based on a social history of limited contact with others after an exposure. ${ }^{10}$ Whether our patient's absence of circulating B cells with subsequent hypogammaglobulinemia predisposed her to a prolonged incubation period is not known. Her negative serology suggests a poor humoral response to infection.

This report has significant implications for preventing the spread of SARS-CoV-2. For patients with known humoral immune deficits, until further data are available, one should exercise caution using a 14-day quarantine window based on the assumption of 14 days being the upper bound of the incubation period. It remains possible that this patient was shedding viable virus from the date of her initial positive test to beyond the date of her bronchoscopy 41 days later. This patient's presymptomatic transmission window may have therefore been substantially greater than the estimated mean presymptomatic transmission window of 2.3 days in the general population. ${ }^{1}$ Whether prolonged incubation periods may occur in other immunosuppressing conditions remains to be evaluated, and further data in this area are needed to better define the appropriate quarantine period in this population.

Supplementary material. To view supplementary material for this article, please visit https://doi.org/10.1017/ice.2020.1239

Acknowledgments. The antibody generated against SARS-CoV 3a protein was a kind gift from Carolyn Machamer, Department of Cell Biology, Johns Hopkins University School of Medicine.

Financial support. J.H. is supported by the Cancer Research Institute Irvington Postdoctoral Fellowship.

Conflicts of interest. The authors report no conflicts of interest.

\section{References}

1. He X, Lau EHY, Wu P, et al. Temporal dynamics in viral shedding and transmissibility of COVID-19. Nat Med 2020;26:672-675.

2. Li Q, Guan X, Wu P, et al. Early transmission dynamics in Wuhan, China, of novel coronavirus-infected pneumonia. N Engl J Med 2020;382:1199-1207. 
3. Lauer SA, Grantz KH, Bi Q, et al. The incubation period of coronavirus disease 2019 (COVID-19) from publicly reported confirmed cases: estimation and application. Ann Intern Med 2020;172:577-582.

4. Backer JA, Klinkenberg D, Wallinga J. Incubation period of 2019 novel coronavirus (2019-nCoV) infections among travellers from Wuhan, China, 20-28 January 2020. Euro Surveill 2020;25(5):2000062.

5. Leung C. The difference in the incubation period of 2019 novel coronavirus (SARS-CoV-2) infection between travelers to Hubei and nontravelers: the need for a longer quarantine period. Infect Control Hosp Epidemiol 2020; 41:594-596.

6. Nie X, Fan L, Mu G, et al. Epidemiological characteristics and incubation period of 7,015 confirmed cases with coronavirus disease 2019 outside Hubei Province in China. J Infect Dis 2020;222:26-33.
7. Linton NM, Kobayashi T, Yang Y, et al. Incubation period and other epidemiological characteristics of 2019 novel coronavirus infections with right truncation: a statistical analysis of publicly available case data. J Clin Med 2020;9(2): 538.

8. Coronavirus disease 2019 (COVID-19) quarantine and isolation. Centers for Disease Control and Prevention website. https://www.cdc.gov/coronavirus/ 2019-ncov/if-you-are-sick/quarantine-isolation.html. Accessed June 6, 2020.

9. Kucirka LM, Lauer SA, Laeyendecker O, Boon D, Lessler J. Variation in false-negative rate of reverse transcriptase polymerase chain reaction-based SARS-CoV-2 tests by time since exposure. Ann Intern Med 2020. doi: 10. 7326/M20-1495.

10. Wang Y, Wang Q, Wang K, Song C, Guo Z, Hu W. A case of COVID-19 with ultra-long incubation period. Infect Control Hosp Epidemiol 2020. doi: 10.1017/ice.2020.221.

\title{
Patients' anxiety, fear, and panic related to coronavirus disease 2019 (COVID-19) and confidence in hospital infection control policy in outpatient departments: A survey from four Thai hospitals
}

\author{
Anucha Apisarnthanarak MD ${ }^{1}$, Chanida Siripraparat MD, MPH², Piyaporn Apisarnthanarak $\mathrm{MD}^{3}$, Michael Ullman $\mathrm{PhD}^{4}$, \\ Pavarat Saengaram MD ${ }^{5}$, Narakorn Leeprechanon $\mathrm{MD}^{6}$ and David J. Weber $\mathrm{MD}^{7}$ \\ ${ }^{1}$ Division of Infectious Diseases, Faculty of Medicine, Thammasat University, Prathum Thani, Thailand, ${ }^{2}$ Manorom Hospital, Bangkok, Thailand, ${ }^{3}$ Division of \\ Diagnostic Radiology, Department of Radiology, Faculty of Medicine Siriraj Hospital, Mahidol University, Bangkok, Thailand, ${ }^{4}$ Research and Consulting Service, \\ Michael Ullmann Consulting, Baltimore, Maryland, United States, ${ }^{5}$ Bumrungrad Hospital, Bangkok, Thailand, ${ }^{6}$ Department of Ophthalmology, Rutnin Hospital, \\ Bangkok, Thailand and ${ }^{7}$ Division of Infectious Diseases, University of North Carolina, Chapel Hill, North Carolina, United States
}

To the Editor-The emergence of the coronavirus disease 2019 (COVID-19) pandemic has disrupted day-to-day patient life with limitations to social practices (eg, physical distancing, mask wearing, and frequent hand hygiene). ${ }^{1}$ These limitations, together with widespread anxiety and stress, have generated a mental health crisis among patients. ${ }^{2}$ Anxiety, fear and panic related to COVID-19 may result in strong emotions and reactions. ${ }^{1-3}$ Therefore, we conducted a survey to evaluate COVID-19associated patient emotions and confidence in hospital infection prevention (IP) and IP behaviors in outpatient departments.

This survey was performed at 2 university hospitals and 2 private hospitals from May 1 to May 30, 2020. To represent multiple patient populations, patients visiting 3 outpatient departments (general medicine, ophthalmology, and radiology) were invited to participate in the study and were interviewed using a standardized data collection tool. The first 50 patients who filled out the survey in each hospital were included in the data analysis. The data collected included patient demographics, perception of risks to contract COVID-19, confidence in policy and preparedness plan for COVID-19, sources of knowledge, and emotions evoked by COVID-19, and IP practices (eg, hand hygiene, wearing a mask, and physical distancing). Respondents rated their confidence level on knowledge and hospital preparedness plan on a scale

Author for correspondence: Anucha Apisarnthanarak, E-mail: anapisarn@yahoo.com Cite this article: Apisarnthanarak A, et al. (2021). Patients' anxiety, fear, and panic related to coronavirus disease 2019 (COVID-19) and confidence in hospital infection control policy in outpatient departments: A survey from four Thai hospitals. Infection Control \& Hospital Epidemiology, 42: 1288-1290, https://doi.org/10.1017/ice.2020.1240 from 1 to 5 (1, "no confidence" to 5 , "very confident") as well as changing in IP behaviors on a scale from 1 to 5 (1, "never use" to 5 , "always use"). IP behavior changes (eg, hand hygiene, wearing a mask, and physical distancing) were defined as a rating of 4 (almost always) or 5 (always). We used the Generalized Anxiety Disorder 7-item (GAD-7) scale to categorize anxiety, self-rated fear, and panic on a scale from 1 to 10 (1, "no fear/panic" to 10 , "extreme fear/panic"). The categorization of the GAD-7 score followed the original scale (ie, 0-4, minimal anxiety; 5-9, mild anxiety; $10-14$, moderate anxiety; and $>14$, severe anxiety), ${ }^{4}$ and self-reported fear $>6$ was categorized as fear of COVID- 19 .

All analyses were performed using SPSS version 19 software (IBM, Armonk, NY). The $\chi^{2}$ or Fisher exact test was used to compare categorical variables. The Mann-Whitney $U$ test was used for continuous data. All $P$ value were 2 -tailed, and $P<.05$ was considered statistically significant. Multivariate analysis was used to evaluate factors associated with emotions and impact of emotions on IP practices.

In total, 200 patients participated in this survey $(n=50$ patients per hospital). The median age of respondents was 45 years (range, $15-92)$, and 138 of 200 participants (70\%) were women. Some patients reported having had contact with COVID-19 patients or a patient under investigation (19 of 200, 9.6\%). Anxiety, fear, and panic related to COVID-19 were reported by 181 of 200 (90\%), 89 of $200(45 \%)$, and 82 of $200(41 \%)$, respectively. Feelings of discrimination and stigma against COVID-19 patients were reported by 113 of $200(57 \%)$ and 107 of $200(54 \%)$, respectively. Social media ( 164 of $200,83 \%$ ) was the most common source

(c) The Author(s), 2020. Published by Cambridge University Press on behalf of The Society for Healthcare Epidemiology of America. This is an Open Access article, distributed under the terms of the Creative Commons Attribution licence (http://creativecommons.org/licenses/by/4.0/), which permits unrestricted re-use, distribution, and reproduction in any medium, provided the original work is properly cited. 